\title{
Em tempos de COVID-19: um olhar para os estudos epidemiológicos observacionais
}

In times of COVID-19: a look at observational epidemiological studies

En el tiempo COVID-19: una mirada a los estudios epidemiológicos

observacionales

Leandro Rozin ${ }^{1}$

${ }^{1}$ Enfermeiro. Mestre em Biotecnologia Aplicada à Saúde da Criança e do Adolescente pelas

Faculdades Pequeno Príncipe. Docente de saúde coletiva e epidemiologia nas Faculdades Pequeno Príncipe. Curitiba, Paraná.

\section{INTRODUÇÃ̃o}

A epidemiologia tem se defrontado com novos desafios a partir do surgimento da COVID-19. A produção de conhecimento em busca de informações seguras e com ritmo correspondente à magnitude e extensão da pandemia, reacendem a relevância dos estudos epidemiológicos entre pesquisadores, profissionais, docentes e estudantes da área da saúde.
Aspecto importante destacado nas Diretrizes Curriculares Nacionais (DCNs) para os cursos na área de saúde é a formação com foco na realidade epidemiológica e nos problemas urgentes vivenciados pela sociedade. Esse aspecto tem por objetivo integralizar as competências para subsidiar o aprendizado e, consequentemente, contribuir para a formação de melhores profissionais para atuarem na complexidade ${ }^{1}$.

Autor de Correspondência:

*Leandro Rozin. E-mail: leandrorozin@hotmail.com 
Diante da situação pandêmica vivenciada no Brasil e no mundo, a atenção no ensino-aprendizado volta-se à busca por conhecimentos que integram a epidemiologia. Por se tratar de uma nova doença, o conhecimento sobre a COVID-19 pode ser subsidiado pelos estudos epidemiológicos.

A epidemiologia se dedica ao estudo da ocorrência de doenças e agravos à saúde nas populações, a fim de definir estratégias de prevenção e controle que podem ser avaliadas também por meio de estudos epidemiológicos. A finalidade dos estudos é conhecer e avaliar os condicionantes e determinantes do processo saúde e doença de populações, de forma geral ou específica. Além de identificar o impacto das ações e insumos propostos e desenvolvidos para intervir no curso de problemas de saúde ${ }^{2-4}$.

Enquanto ciência, a epidemiologia integra métodos e técnicas que incorpora outras três áreas de conhecimento: Estatística, Ciências da Saúde e Ciências Sociais. Sua área compreende tanto o ensino quanto a pesquisa em saúde, avaliação de procedimentos e serviços de saúde, vigilância epidemiológica e monitoramento da situação de saúde das populações. Enquanto o foco da clínica é o indivíduo, a epidemiologia atua com grupos populacionais, tornando insubstituível a abordagem quantitativa em seus estudos ${ }^{2,5-7}$.

Embora os estudos epidemiológicos tenham sido desenvolvidos antes do século XX, a sistematização de princípios que possibilitassem o seu delineamento e avaliação estruturou-se com mais clareza na segunda metade daquele século. Esses princípios evoluíram com uma explosão de pesquisas epidemiológicas com diferenciadas tipologias e seu progresso continua até hoje. Em alguns casos, a evolução conceitual prática e controversa levou a diferentes interpretações e à condução dessas pesquisas ${ }^{5,8-9}$.

Apesar da crescente utilização dos estudos, sua classificação conceitual e aplicabilidade permanecem em constante estágio de desenvolvimento. Isso reflete a preocupação sobre a conformidade de utilização e validade dos métodos selecionados e, por consequência, na confiabilidade dos resultados ${ }^{2,7,9}$.

Para a realização de um estudo epidemiológico, é importante seguir as etapas: 1. Determinar os objetivos; 2. Organizar o delineamento adequado conforme a viabilidade do estudo e os recursos disponíveis; 3. Definir a população de estudo; 4. Planejar a condução da pesquisa; 5. Sistematizar a coleta, análise e interpretação dos dados; e, 6. Apresentar os resultados ${ }^{10}$. Para garantir a qualidade, é necessário, entre outros fatores, estar atento para a representação dos participantes e da qualidade dos dados ${ }^{4}$. Os instrumentos de coleta de dados quando não validados devem ser pré-testados, tendo como panorama os dados ou medidas que se deseja obter. Além disso, é necessário atentar-se aos aspectos éticos pertinentes, com base nas normas e resoluções existentes para pesquisas com seres humanos ${ }^{10-12}$.

Baseada em pesquisas e evidências científicas, atualmente os métodos padrão de análise se baseiam em erros aleatórios e cálculos de significância, como desvios-padrão, valores $\mathrm{P}$, intervalos de confiança e testes de hipótese. A análise dos estudos observacionais, especificamente nos analíticos, se baseia em alguns cálculos de razões específicas a cada tipo de estudo, e podem ser confirmados por testes padrões de erros aleatórios ${ }^{13-15}$.

Os estudos epidemiológicos se classificam em observacional e experimental. Os observacionais são comumente utilizados em uma investigação epidemiológica para descrever ou determinar a ocorrência de uma doença ou agravo à saúde em uma população a partir de um ou mais grupos classificados no início do estudo. Nesses estudos não há intervenção do pesquisador em fatores que determinam ou condicionam o processo que leva ao agravo ou à doença, ou que possa alterar seu percurso natural ${ }^{4,8,12-15}$

Já nos estudos experimentais, ocorre a intervenção 
direta do pesquisador com a relação analisada, havendo a possibilidade de controle de fatores selecionados, residindo aí a importância desse processo. Inclui os ensaios clínicos e ensaios de campo cujo foco de estudo é o indivíduo, ou os ensaios de intervenção comunitária cuja unidade de estudo é um agregado populacional ${ }^{8,12,14-15}$.

A divergência conceitual, somada à carência de publicações recentes que discutam os métodos de estudos epidemiológicos, especialmente no tipo observacional, é claramente percebida entre pesquisadores e estudantes que buscam definir, a partir de seus objetivos, o estudo adequado a ser utilizado e a garantia da confiabilidade dos resultados.

Em tempos de pandemia de COVID-19, observase que a procura pelos estudos epidemiológicos tem aumentado consideravelmente para ampliar o conhecimento a respeito da doença. Portanto, a presente discussão é resultante de um ensaio teórico reflexivo cujo propósito é oferecer subsídios que permitam a conformidade de utilização dos estudos epidemiológicos observacionais para o ensino e a pesquisa, no atual cenário de saúde.

\section{ESTUDOS EPIDEMIOLÓGICOS OBSERVACIONAIS: CONCEITOS E CLASSIFICAÇÃO}

Os estudos observacionais podem ser classificados em descritivos ou analíticos (Figura 1).

Os estudos descritivos são realizados quando não existe grupo de comparação. Portanto, quando não se objetiva a análise da associação entre exposição da doença ou agravo com o desfecho. Dessa forma, são utilizados para a elaboração de hipóteses que podem ser posteriormente complementadas e enriquecidas pelos estudos analíticos ${ }^{4-6,8,13-14}$.
Os estudos epidemiológicos descritivos associamse diretamente à linha da epidemiologia descritiva, que enfatiza a compreensão do comportamento de uma doença ou agravo à saúde de uma população, explorando características da pessoa, tempo e lugar, respondendo as questões: Quem? Quando? Onde? Os tipos levantamento e inquérito epidemiológico, estudo de caso ou de série de casos fazem parte dos estudos observacionais descritivos. Assim como os estudos ecológicos, apesar de também serem classificados como analíticos ${ }^{7,9,12-13}$.

Nos estudos analíticos, o grupo de comparação deve estar presente para medir associação e hipóteses. Permitem a identificação de associações entre doenças ou agravos e diversos fatores que podem ter importância no evento e/ou em seu desfecho, medindo associação de hipóteses elaboradas durante os estudos descritivos. Fazem parte dos estudos observacionais analíticos os tipos ecológico, transversal, caso-controle e coorte $e^{4,7,9,14-15}$.

Portanto, os estudos descritivos expõem perfis populacionais, de grupos ou de um caso, podendo fazer uso de taxas ou proporções que melhor apresentem e comparem os resultados. Pertencem também ao descritivo, os estudos ecológicos que originam hipóteses sem medir associação, porém com características comparativas peculiares. Já os estudos analíticos, utilizam taxas ou coeficientes epidemiológicos para testar associações por meio de razões determinadas em cada estudo. Geralmente as medidas de associação por teste de hipóteses entre causa e efeito, declaradas na ocasião do planejamento do estudo e analisadas por meio da associação, são complementadas por testes estatísticos ${ }^{6-9,13-15}$. 
Figura 1 - Classificação dos estudos epidemiológicos observacionais com as principais peculiaridades classificatórias.

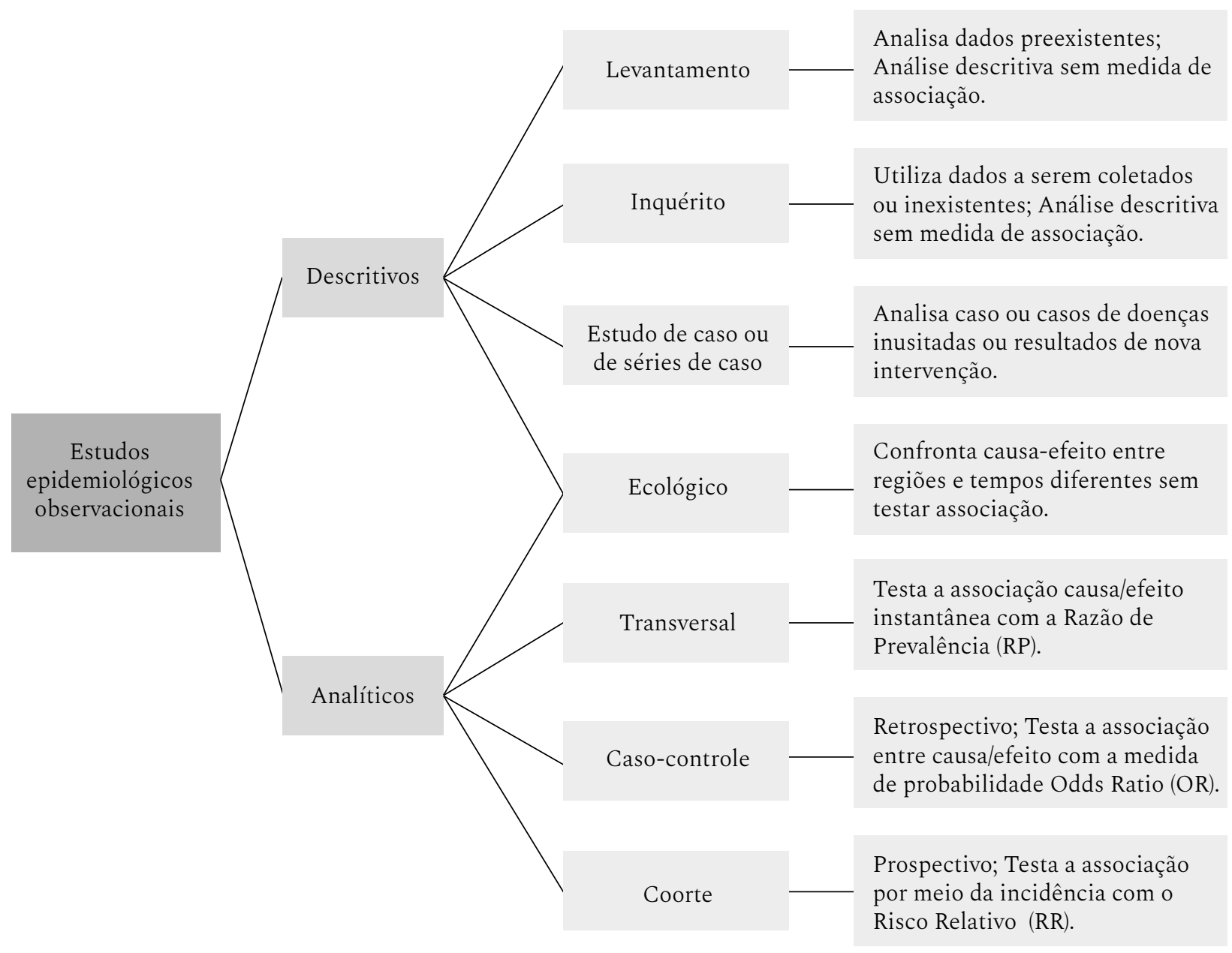

Fonte: autor, 2020.

\section{LEVANTAMENTO EPIDEMIOLÓGICO}

O levantamento epidemiológico apresenta papel relevante na pesquisa em saúde por explorar o perfil epidemiológico a partir de dados preexistentes de base documental. Compõe a primeira etapa da aplicação do método epidemiológico, com vistas à compreensão do comportamento de um agravo à saúde em uma população, a partir da coleta de dados preexistentes, geralmente disponíveis em Sistemas de Informação em Saúde (SIS). A pesquisa busca investigar as questões relativas ao quem, quando e onde? Ou seja, apresentar as características epidemiológicas relacionadas a pessoa, tempo e lugar ${ }^{4,16-17}$

A descrição da investigação epidemiológica está relacionada às características referentes à pessoa, especialmente idade, sexo, escolaridade, nível socioeconômico, etnia, ocupação, situação conjugal, usuário ou não de serviços de saúde, com ou sem saneamento básico, etc. Busca responder quem é o indivíduo acometido pela doença ou agravo ${ }^{5,7,9,17}$. 
Com relação ao tempo, enfatiza-se o comportamento das doenças em diferentes períodos, com o objetivo de caracterizar tendências, variações regulares. Por exemplo, as variações cíclicas e sazonais ou as variações irregulares que podem caracterizar epidemias. Também é possível analisar por meio de levantamento de série histórica, com análise de dados anuais de no mínimo 10 anos. Por sua vez, a descrição relativa ao lugar preocupa-se com aspectos da distribuição geográfica que deve estar bem delimitada, apresentando as diferenças do comportamento das doenças ou agravos conforme territórios ou distintas regiões do mundo ou de comunidades. As escolhas das variáveis dependem dos objetivos do estudo ${ }^{5,7,9,17}$.

Para esse tipo de estudo, utiliza-se com frequência a estatística descritiva com uso da distribuição proporcional, cálculos de taxas de indicadores de saúde como, por exemplo, a prevalência, incidência, letalidade, entre outras. Apesar da análise simples, pode ser enriquecida com testes estatísticos que se baseiam em erros aleatórios, como desviospadrão, valores $\mathrm{P}$, intervalos de confiança e testes de hipótese $\mathrm{5}^{5,7,9,17}$.

Um exemplo para tal estudo é quando se busca identificar o perfil epidemiológico de gestantes com sífilis em um local e em um período preestabelecido. Outro, em tempos de pandemia, é determinar o perfil epidemiológico da COVID-19. Em ambos os exemplos, a fonte de dados proverá do Sistema de Informações de Agravos de Notificação (SINAN). Por se tratar de uma doença de notificação, com instrumento padrão e constantemente preenchido, os dados com diversas variáveis estão disponíveis no Departamento de Vigilância Epidemiológica. base de dados utilizada, com dados inexistentes ou insuficientes em outras fontes, que devem ser coletados por meio de instrumentos validados ou não. Por se tratar da definição por meio de instrumentos validados ou da criação de instrumento com as variáveis a serem coletadas, os inquéritos possibilitam a obtenção de informações sobre diferentes variáveis de interesse relacionadas à saúde que também descrevem um perfil epidemiológico. Os dados coletados têm base nos determinantes demográficos, sociais, econômicos e culturais da saúde-doença, além de comportamentos relacionados à saúde, muitos dos quais constituem fatores de risco de doenças e agravos à saúde $\mathrm{e}^{11,17-18}$.

Esse tipo de estudo, aplicado às condições de saúde de populações, têm sido cada vez mais utilizados no Brasil, especialmente na pesquisa de doenças não transmissíveis como componente para a geração de informações necessárias à formulação e avaliação das políticas de promoção de saúde e de prevenção e controle de agravos. Os instrumentos de medida são geralmente registros, questionários, exames clínicos e testes de laboratório previamente validados ou construídos pelo pesquisador quando as informações existentes são inadequadas ou insuficientes ${ }^{17-18}$.

Um exemplo desse estudo é uma pesquisa de identificação de perfil de adolescentes que fazem uso abusivo de álcool. Como não existem dados prévios em banco de dados, o pesquisador precisará selecionar um instrumento validado ou construir um instrumento para coletar os dados das variáveis com suficiência para alcançar o objetivo da pesquisa. Assim como nos estudos do tipo levantamento, o inquérito também se apoia na epidemiologia descritiva e busca conhecer a pessoa, tempo e lugar para o alcance do objetivo do estudo.

\section{ESTUDO DE CASO E DE SÉRIES DE CASO}

Os estudos de caso ou de séries de caso objetivam 
o detalhamento de um ou vários casos clínicos que apresentem características pouco frequentes de uma nova doença, de doença já conhecida com novos desfechos ou de uma nova intervenção. Para a série de casos, recomenda-se que o grupo analisado seja de no mínimo 10 casos. Geralmente esses estudos visam a atenção de outros pesquisadores que tenham efetuado pesquisas similares, criando condições para formulações de hipóteses e possibilidades para outros tipos de estudos ${ }^{6,19-21}$.

A análise de um caso excepcional pode não caracterizar um estudo epidemiológico. Isso se justifica em virtude da análise ser individualizada e não coletiva, mas é o ponto de partida para novas investigações epidemiológicas. Já na análise de série de vários casos incomuns, os resultados possibilitam melhores hipóteses, mesmo consideradas subjetivas. Dessa forma, os estudos de caso ou de série de casos tomam posições hierarquicamente inferiores quanto ao nível de evidência de um estudo ${ }^{21-22}$. Embora tenham um nível de evidência baixo, deram origem a inúmeras investigações relevantes como quando surgiu a AIDS e nos casos iniciais que deram base aos estudos da COVID-19.

Muitas vezes é utilizada a descrição de uma série de casos para caracterizar riscos de padrões irregulares de uma doença e até emergência como uma epidemia. Na série de casos há riscos de conclusões equivocadas já que avaliam ocorrências do passado e os resultados são aplicados apenas àqueles participantes e não à coletividade $^{6,19-21}$.

\section{ESTUDO ECOLÓGICO}

Alguns autores classificam os estudos ecológicos como descritivos, por não haver teste específico para validar a associação de hipóteses. Outros os consideram melhor classificados como analíticos, por examinarem a existência de associação entre a exposição e uma doença ou agravo relacionados à saúde ${ }^{2,5,8,10}$.
No estudo ecológico compara-se a associação entre uma doença ou condição de saúde e a exposição à causa com outras populações, geograficamente definidas e comparáveis (países, regiões, municípios e bairros, por exemplo) e com comparações de temporalidade. Seu objetivo é verificar a hipótese de existência de associação entre elas, porém sem um teste específico para comprovar a associação $0^{4,10,12}$.

Os estudos ecológicos são bem utilizados para gerar hipóteses que dão suporte a outras pesquisas analíticas que testam essas hipóteses. O diferencial dos estudos ecológicos está na análise de associação comparativa entre locais diferentes, que sugerem ou não que uma causa está relacionada a um agravo ou doença/óbitos. Além da comparativa de diferentes locais, a análise inclui diferentes períodos de tempo, em uma série temporal, comparando-se a mesma população em diferentes momentos ${ }^{9-10,12-15}$.

Um exemplo é um estudo envolvendo cidades, em que se procura correlacionar dados sobre mortalidade infantil com a renda do local, a fim de verificar se a associação de que o nível socioeconômico é um determinante da mortalidade infantil. Outro seria correlacionar o consumo de gordura com o câncer de mama entre diversos países com consumo alimentar diverso.

\section{TRANSVERSAL}

Os estudos transversais, também chamados de seccionais, medem a prevalência da doença por meio da associação da causa e efeito (doença) de forma momentânea. Esse estudo é erroneamente confundido em muitas pesquisas com o estudo descritivo do levantamento epidemiológico. O que caracteriza um estudo transversal é o teste da associação causa/efeito com Razão de Prevalência (RP). Um exemplo para esse tipo de estudo é testar a hipótese de que o fumo está relacionado ao câncer ou testar a hipótese de associação entre a obesidade 
como risco para hipertensão, 5,8,16,19-20.

O desenho da pesquisa está na seleção de uma população e dela, de forma aleatória, compor uma amostra. A partir dessa amostra, são identificados 4 grupos: 1) doentes e expostos à causa analisada; 2) doentes e não expostos à causa; 3) não doentes e expostos à causa; 4) não doentes e não expostos à causa. Assim, calcula-se a prevalência dos grupos de doentes e expostos (1) e dos doentes e não expostos (2). A taxa de prevalência é o resultante do número de casos da doença divididos pelo total da população exposta ao risco. A partir disso, calculase a Razão de Prevalência (RP), que é a divisão do resultado da prevalência de doentes e expostos (1) por doentes e não expostos (2). O resultado evidencia se há associação entre doença e causa, interpretada pelos resultados: $\mathrm{RP}=1,0$ - Não há associação; RP > - 1,0 Associação Positiva; RP < 1,0 - Associação Negativa ${ }^{8-9,14-15}$.

O objetivo é a identificação de aspectos relativos à etiologia da doença. Assim, são particularmente indicados para investigar fatores de risco de doenças de início lento e de evolução longa. São muito utilizadas para determinar fatores de risco para doenças crônicas e têm sido conduzidas em um grande número de países. As pesquisas podem ser realizadas no mesmo local, e de maneira regular, com uma amostra representativa para acompanhar a situação de uma doença em relação à evolução das causas. Essas pesquisas enfatizam características pessoais e demográficas, sexo, grupo étnico e hábitos relativos à saúde $\mathrm{e}^{16,20-21}$.

\section{CASO CONTROLE}

Nos estudos de caso controle, parte-se do "efeito" (doença ou agravo) para identificar as causas, a exposição ao risco. Ou seja, a pesquisa busca compreender a etiologia de forma retrospectiva. Assim, a pesquisa é realizada a partir do fato consumado, questionando-se: Quais são as causas do agravo à saúde? ${ }^{10,15,19}$.

Para a sua realização, identifica-se inicialmente a população a ser estudada e seleciona-se dois grupos de mesmo número, com características semelhantes. Indivíduos com a doença ou o agravo são os casos e, para comparação, o mesmo número de indivíduos sem a doença ou o agravo, que são o controle. Após a seleção, aplica-se a Odds Ratio (OR) da exposição entre casos. "Odds Ratio (OR)" é um outro tipo de medida de efeito que significa a razão ou relação de chances ou probabilidades ${ }^{3,9,13,16}$.

Para o cálculo de OR, dividem-se os casos (a) doentes e expostos ao fator de risco por (c) doentes e não expostos, assim como o controle: (b) não doentes e expostos por (d) não doentes e expostos. Com o resultados desses dois cálculos, divide-se os resultados de ac por bd, resultando no Odds Ratio (OR). Se existir associação entre a exposição e a doença, espera-se que a Odds da exposição entre casos seja maior que a observada entre controles, além da variação esperada, que se deve ao acaso ${ }^{19-20,23}$.

Sua interpretação é: $\mathrm{OR}<1$ indica uma associação "protetora”, diminuindo o risco (chance) de doença entre os expostos. $\mathrm{OR}=1$ indica que não há associação entre ambas as variáveis, a associação é improvável. $\mathrm{OR}>1$ indica que há uma associação, sendo mais forte quanto maior for o número, ou seja, aumenta o risco (chance) de doença entre os expostos ${ }^{14-15,20,23}$.

Um exemplo de estudo caso-controle é avaliar a associação entre Infecção Sexualmente Transmissível (IST) com o uso de drogas, observando retrospectivamente indivíduos com IST se o uso de drogas foi um fator de risco para a doença.

\section{COORTE}

Nesse tipo de estudo, também chamado de estudo longitudinal ou de incidência, os desfechos (o 
surgimento da doença ou não) são comparados a partir da exposição de forma prospectiva, a fim de conhecer a história natural da doença ou agravo estudado. Busca identificar em longo prazo o que o estudo transversal realiza de maneira imediata, porém com base na incidência e não na prevalência da doença, aumentando a confiabilidade da pesquisa $^{12,13,16}$.

Portanto, objetiva acompanhar a incidência da doença nos diferentes grupos que são transformados em subgrupo de expostos a um determinado fator de risco se comparada com o subgrupo não exposto, buscando, em outros termos, coligar se há associação entre a exposição e desfecho de interesse ${ }^{19,23}$.

Um estudo de coorte é formado por um ou mais grupos de pessoas sadias ou que ainda não apresentam o desfecho a ser investigado, selecionadas por apresentarem características semelhantes. Esses participantes são classificados em subgrupos ao longo do tempo, após a exposição ou não ao fator associado à ocorrência da doença ou agravo de investigação. Dessa forma, a cada período analisado, formam-se os subgrupos que se classificam em expostos e não expostos a um fator de risco com o surgimento ou não da doença ou agravo estudado. Os subgrupos não precisam ter números iguais, pois variam conforme a evolução da pesquisa $a^{8,10,13,23-24}$.

Na sequência, os indivíduos de ambos os grupos são acompanhados em períodos de tempo determinados pelo pesquisador (mensal ou anual, por exemplo), calculando a taxa de incidência em cada período determinado. Assim, utiliza-se os subgrupos (a) expostos ao risco e que desenvolveram a doença, (b) expostos ao risco que não desenvolveram a doença, (c) não expostos ao risco que desenvolveram a doença e, (d) não expostos e que não desenvolveram a doença. Calcula-se a taxa de incidência do grupo de exposto $(\mathrm{a} / \mathrm{a}+\mathrm{b})$ e do não exposto $(\mathrm{c} / \mathrm{c}+\mathrm{d})$. A taxa de incidência é calculada utilizando o número de casos novos da doença ou agravo, divididos pela população exposta ao risco ${ }^{4,13,23-24}$.

A partir das taxas de incidência de cada grupo, calcula-se o Risco Relativo (RR), baseado na razão de ocorrência. O RR Indica quantas vezes maior é o risco de adoecer entre os expostos comparados aos não expostos. O cálculo é a razão entre duas taxas de incidência, ou seja, divide-se a taxa de incidência do grupo exposto dividido pela taxa do grupo não exposto $0^{8,10,13}$.

Um RR menor ou igual a 1 indica que a incidência do desfecho foi igual nos dois grupos comparados, ou seja, a exposição a determinado fator não causou maior risco de doença nos expostos, comparativamente aos não expostos. Um RR maior que 1, significa associação positiva. Quanto maior o número, maior a força da associação entre exposição e doença ${ }^{4,23-24}$.

Esse ensaio reflexivo esclarece objetivamente alguns aspectos conceituais da epidemiologia e do delineamento de estudos epidemiológicos observacionais que podem ser utilizados para as pesquisas de investigação de doenças e fatores associados a elas na coletividade. Destacase a carência de publicações que debatam claramente a aplicabilidade desses métodos para o desenvolvimento de pesquisas.

Assim, acredita-se que a presente reflexão contribui com subsídios para estudantes, pesquisadores e profissionais da saúde que buscam compreender a adequada aplicabilidade dos estudos epidemiológicos observacionais em tempos de pandemia de uma nova doença, a ser ainda amplamente estudada.

\section{REFERÊNCIAS}

1. Cyrino AP, Godoy D, Cyrino EG. Saúde, ensino e comunidade: reflexões sobre práticas de ensino na atenção primária à saúde. São Paulo: Cultura Acadêmica; 2014.

2. Rouquayrol ZM, Almeida-Filho N. Epidemiolo- 
gia e Saúde. 6. ed. Rio de Janeiro: Guanabara Koogan; 2009.

3. Medronho RA, Bloch KV, Luiz RR, Werneck GL. Epidemiologia. 2. ed. São Paulo: Atheneu; 2009.

4. Brasil. Ministério da Saúde. Guia para Investigações de Surtos ou Epidemias. Brasília: Ministério da Saúde; 2018.

5. Rouquayrol MZ, Guergel M. Epidemiologia e saúde. 7. ed. Rio de Janeiro: MedBook; 2013.

6. Bonita R, Beaglehole R, Kjellström T. Epidemiologia básica. [livro online] 2. ed. São Paulo: Santos, 2010. [Acesso em 10 abr 2020]. Disponível em: https://apps.who.int/iris/ bitstream/handle/10665/43541/9788572888394_por.pdf?sequence $=5 \&$ is Allowed $=y$

7. Rothman K, Greenland S, Lash TL. Epidemiologia moderna. 3. ed. Porto Alegre: Artmed; 2011.

8. Gomes ECS. Conceitos e ferramentas da epidemiologia. [livro online] Recife: Ed. Universitária da UFPE; 2015. [Acesso em 10 abr 2020]. Disponível em: https://ares.unasus.gov.br/assetsto re/37/52/69/37526977859140716131665198799384657433

9. Pereira MG. Epidemiologia: teoria e prática. Rio de Janeiro: Guanabara Koogan; 2013.

10. Lima-Costa MF, Barreto SM. Tipos de estudos epidemiológicos: conceitos básicos e aplicações na área do envelhecimento. Epidemiol Serv Saúde. 2003;12(4):189-201. [Acesso em 15 abr 2020]. Disponível em: http://dx.doi. org/10.5123/S1679-49742003000400003.

11. Andrade FR, Narvai PC, Montagner MA. Implicações éticas de inquéritos populacionais epidemiológicos. Physis. 2017; 27(3):475-489. [Acesso em 15 abr 2020]. Disponível em: https://scielosp.org/article/physis/2017.v27n3/475489 /

12. Almeida Filho N, Baretto ML. Epidemiologia \& saúde: fundamentos, métodos e aplicações. Rio de Janeiro: Guanabara Koogan; 2011.

13. Fronteira I. Estudos observacionais na Era da Medicina baseada na evidência: breve revisão sobre a sua Relevância, Taxonomia e Desenhos. Acta Med Port. 2013;26(2):161-70. [Acesso em 18 abr de 2020]. Disponível em: file://C:/Users/
Cliente/Downloads/3975-5347-1-PB.pdf

14. Malta M, Cardoso LO, Bastos FI, Magnanini MMF, Passos da Silva CMF. Iniciativa STROBE: subsídios para a comunicação de estudos observacionais. Rev Saúde Pública. 2010;44(3):559-65. [Acesso em 18 abr de 2020]. Disponível em: https://www.paho.org/revelac-i/wp-content/ uploads/2016/03/STROBE_translation_portuguese_Commentary_Malta_RevSaudePublica_2010_checklist.pdf

15. Aragão J. Introdução aos estudos quantitativos utilizados em pesquisas científicas. Revista Práxis. 2011;3(6). [Acesso em 18 abr de 2020]. Disponível em: http://web. unifoa.edu.br/praxis/numeros/06/59.pdf . Acesso em 05/05/2018.

16. Porta M, Greenland S, Last J. A dictionary of Epidemiology. 6. ed. Oxford: Oxford University Press; 2014. [Acesso em 18 abr de 2020]. Disponível em: https://books. google.com.br/books?hl=it\&lr=\&id=vEZmAwAAQBA$\mathrm{J} \&$ oi=fnd\&pg=PP1\&ots=ei8NlGMvX8\&sig=78rT1a1LIYc-LXqK92x-m4aip7E\&redir_esc=y\#v=onepage\&q\&f=false

17. Brasil. Ministério da Saúde. Secretaria de Vigilância em Saúde. Departamento de Vigilância Epidemiológica. Guia de vigilância epidemiológica. 7. ed. Brasília: Ministério da Saúde; 2009. [Acesso em 15 abr de 2020]. Disponível em: https://bvsms.saude.gov.br/bvs/publicacoes/guia_vigilancia_epidemiologica_7ed.pdf

18. Barros MBA. Inquéritos domiciliares de saúde: potencialidades e desafios. Rev Bras Epidemiol. 2008;11(1):619. [Acesso em 20 abr 2020]. Disponível em: https://doi. org/10.1590/S1415-790X2008000500002.

19. Gordis, L. Epidemiolog. 3. ed. Philadelfia: Sauders Company; 2009.

20. Lopes AC. Tratado de Clínica Médica. 3. ed. São Paulo: Guanabara Koogan; 2015.

21. Yin RK. Estudo de caso: planejamento e métodos. Porto Alegre: Bookman; 2010.

22. Oliveira MAP, Velarde GC, Sá RAM. Entendendo a pesquisa clínica V: relatos e séries de casos. FEMINA. 2015;43(5). [Acesso em 20 abr 2020]. Disponível em: http:// 
files.bvs.br/upload/S/0100-7254/2015/v43n5/a5320.pdf

23. Martins AAB, Teixeira D, Batista BG, Steffens D. Epidemiologia. Porto Alegre: SAGAH; 2018.

24. Hulley SB, Cummings SR, Browner WS, Grady D, Hearst N, Newman TB. Delineando a pesquisa clínica. Uma abordagem epidemiológica. 3. ed. Porto Alegre: Artmed; 2007. 